\title{
SymAB: Symbol-Based Address Book for the Semi-literate Mobile User
}

\author{
Anuradha Bhamidipaty and Deepak P. \\ IBM India Research Lab, Bangalore, India \\ \{abhamidi, deepak.s.p\}@in.ibm.com
}

\begin{abstract}
Developing countries like India are observing an increasing trend in the penetration of mobile phones towards the base of the pyramid (lower strata of the society). This segment comprises of users who are novice and semiliterate and are interested in the basic usage of the mobile phone. This paper explores one of the basic features, the address book for its usability and presents an enhanced symbol-based design to cater for the semi-literate user. The enhancement uses symbols to replace current text based storage and retrieval and also includes a call distribution based address book access to align with the skewed nature of the user's requirements. The results of a preliminary evaluation of the prototype are encouraging regarding the value perceived through the design.
\end{abstract}

\section{Introduction}

Developing countries like India and China have seen a vast growth in the mobile user base with increasing mobile penetration to the lower (lower income) strata of the society. In countries like India where the majority of the population live in rural areas, increasing penetration of mobile devices into the lower strata of the society is critical to insulating the rural population from the vagaries of the highly inefficient and information-asymmetric markets, marked in particular by the tremendous uncertainty and risk of doing business. Mobile devices have started to benefit the poorest of the poor - a study confirming that increased stability of markets is among the effects of the usage of mobile devices [1], [2].

Mobile devices such as mobile phones as existing today assume a reasonable amount of literacy. Although different features provided with the modern mobile devices require different levels of literacy and familiarity with technology, the basic usage of the mobile phone, which is of being able to make and receive calls, requires the ability to understand numerals. The other features that are considered as "basic" features include the address book and the short messaging system (SMS). The former enables the user to call by name and display calls from known users by showing up their name, whereas the latter allows the user to send and receive text messages. Both these features require knowledge of the alphabet; the ability to read and type alphabet. This need is quite intuitive in the short messaging system, text being the most common and uniform mode of information exchange among multiple people. On the other hand, the address book is a personal and local entity, which enables storage and 
retrieval by the user of the mobile device on the device itself. The design of the address book as a text based storage and retrieval interface causes a barrier to it's usage in the growing class of semi-literate mobile users; the class of users who can manage to understand numerals, but cannot read or write alphabets.

Further, people in the said category often have a small set of contacts who are contacted very frequently, whereas a large majority of their contacts are used rarely. The uniform search interface, which roughly requires uniform number of clicks to reach to any contact in the address book, is intuitively unsuitable for the highly skewed access requirements, arising out of the skewed call distribution of the semiliterate mobile user. In this paper, we investigate the usability of the current text based storage and retrieval system that common address books utilize among the class of semi-literate mobile users, and evaluate the effectiveness of an enhanced design for the address book, the $\operatorname{Sym} A B$. We also show that a call distribution based address book access mechanism aligns well with the access requirements of the semi-literate mobile user.

\subsection{Text Usage and the Semi-literate Mobile User}

A survey was conducted among the semi-literate mobile users from the low-income group in the urban area of Bangalore with 20 participants, with ages ranging from 20 to 35 engaged in a variety of manual trades such as cleaners, plumbers, drivers etc. The largely textual interfaces of the mobile devices affected the usage patterns for the semi-literate. Firstly, there is a psychological barrier to the usage of mobile phones as the semi-literate perceives it to be a high-end device, partly because of the textual content involved. Secondly, the semi-literate who realizes the value-add that mobile devices can cause to his work/life and chooses to use it, tends to use it minimally. We refer to minimal usage as the usage of a very small subset of the features available in the device.

Further investigations revealed that the literacy barrier leads to effects including things such as:

- Maintaining address books of essential contacts on sheets of paper, which are looked up when a call needs to be made.

- Seeking the assistance of a literate person to update the address book.

- Taking help from a literate person to store essential numbers into the address book and to learn a sequence of keystrokes to call commonly used numbers such as home, spouse, employer etc.

From our observations, we find that address books are indispensable for mobile usage since every user maintains it, either using the address book on the mobile or using one of the above methods. These scenarios motivate the need to explore the usability of alternate interfaces for illiterate users.

The usage pattern of mobile devices is usually much skewed for the semi-literate. They typically have a small set of contacts that they use frequently (including the employer, family and the inner ring of friends), whereas the rest of the contacts are used very rarely. Although this is true for the most mobile users, regardless of their income and literacy, we argue that the need to address this issue is more critical for the semi-literate due to two factors - (1) Accessing the address book is hard for the 
semi-literate making usability a critical requirement, and (2) the frequently used contacts is a much smaller set in the case of the semi-literate mobile user as compared to the more general categories.

\section{$2 \operatorname{Sym} A B$ Design}

To address the issues highlighted in the survey, we propose an enhanced design of the address book, the $\operatorname{Sym} A B$ which is a symbol-based interface. Symbols have been shown to be very highly preferred over textual interfaces, by the communities that we address [2].

The design composes of two parts, an enhanced mobile keypad with provision of symbols either in addition or as replacement to alphabet and a symbol based retrieval mechanism of the stored entries. A keypad button is associated with a symbol, clicking a button once will enter the symbol and subsequent clicks will bring up the other alphanumeric characters associated with it. We studied the requirements of a sample of semi-literate mobile users in Bangalore and arrived at a small set of symbols to be embedded in the 12-key mobile keypads, the trade-off being to include as much of the symbols in the set suggested by most people, at the same time trying to leave as many slots open so as to allow room for customizability i.e., these slots would be filled with generic symbols allowing users to assign their own meanings to them. The icons chosen were those of a home, family, money, man, mobile, woman, elder-man and elder-woman. The rest of the four icons were optionally populated from the following geometric shapes - square, circle, rectangle, rhombus, triangle and pentagon. A contact can be associated with variable length strings, each element in the string being a symbol, numeric or an alphabet. As is obvious, strings that are purely composed of symbols are assumed to be unordered, i.e., $\triangle$ is assumed to be the same as $\triangle$.

The second part is the symbol-based search functionality for the address book, which is based on a prefix-search, i.e., searching all matches for a prefix entered, is
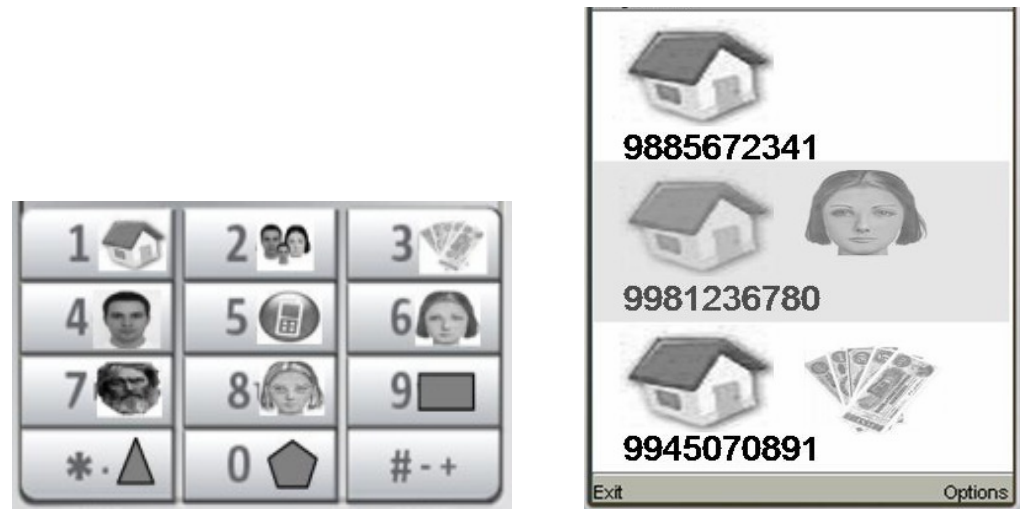

Fig. 1. The design of the keypad (on the left) and the symbol-based search of the address book (on the right) of $S y m A B$ application 
replaced by a subset-search in the $\operatorname{Sym} A B$ interface wherein all contacts represented by supersets of the entered set of symbols is displayed. Figure 1 demonstrates the retrieval of all entries that contain the home symbol. Thus the icons on the keypad are used to limit the search for an entry.

In addition to the above, $\operatorname{Sym} A B$ is further augmented by a call distribution based access which can be accessed by pressing "\#”. SymAB maintains an internal list of contacts sorted in the decreasing order of frequency of calling, $k$ key-presses of the \# key leading to the $k^{\text {th }}$ frequently contacted contact in the address book. This feature is aligned with the skewed call distribution of a typical user in semi-literate category thus helping the user quickly locate the most frequent contact.

\section{Evaluation and Future Work}

We conducted a preliminary evaluation of $\operatorname{Sym} A B$ with 10 participants to obtain qualitative feedback on the value perceived by the enhanced design. All participants belonged to the semi-literate category, with ages ranging from 19 to 28 years. We educated the participants on the use of the $\operatorname{SymAB}$ interface and asked them to simulate the storage of a minimum of 3 contacts and randomly retrieve them. We also asked them questions to get an idea of the skew in their call distribution, and as to how much the frequency based retrieval would be able to help them.

All the users reported the enhanced value they perceived from the design thus making the address book feature simpler, easy-to-use and more comfortable to play around with. They not only utilized the pre-defined set of icons but created combinations of symbols for storing entries. Some of them suggested additional icons to be included, that of a friend, rose and vehicle. The participants also expressed that using $\operatorname{Sym} A B$ their near and dear ones like elderly parents, relatives staying away from them who are semi-literate and not working would now be able contact them for any emergency/need without assistance thus making them independent. One participant indicated that since $S y m A B$ gives co-existence of both icon and text-based storage/retrieval mechanisms, it is multi-purpose serving the needs of both the educated and semi-literate in a family. Most of the participants reported a high skew in call distribution, with the number of contacts used on a daily basis being as low as 2-8 with an average of 4 . All of them found frequency based retrieval as a useful feature, and many opined that it is easier to educate the elderly to retrieve based on such an interface than any other retrieval mechanism.

We are currently exploring the usability of other features of the mobile phone for the semi-literate class and investigating the effectiveness of alternate interfaces.

\section{References}

1. Abraham, Reuben: Mobile Phones and Economic Development: Evidence from the fishing industry. In: India, International Conference on ICT for (ICTD) (May 2006)

2. Waverman, L., Meschi, M., Fuss, M.: The impact of telecoms on economic growth in developing countries, http://web.si.umich.edu/tprc/papers/2005/450/L\%20Waverman\%20Telecoms\%20Growth\%20in\%20Dev.\%20Countries.pdf

3. Medhi, I., Sagar, A., Toyama, K.: Text-free User Interfaces for Illiterate and Semi-Literate Users. In: International Conference on ICT for Development (ICTD) (May 2006) 